Bull. Chem. Soc. Ethiop. 2017, 31(2), 345-350.

ISSN 1011-3924

(c) 2017 Chemical Society of Ethiopia and The Authors

Printed in Ethiopia

DOI: http://dx.doi.org/10.4314/bcse.v31i2.16

\title{
SYNTHESIS AND CHARACTERIZATION OF FUNCTIONALIZED DIHYDROPYRIMIDINONES VIA ONE-POT ISOCYANIDE-BASED THREE-COMPONENT REACTION OF N-FORMYL UREA AND DIALKYL ACETYLENEDICARBOXYLATES
}

\author{
Ainaz Naderi and Bita Mohtat ${ }^{*}$ \\ Department of Chemistry, Islamic Azad University, Karaj Branch, Karaj, Iran
}

(Received July 30, 2016; revised September 11, 2017)

\begin{abstract}
The reactive intermediate generated by the addition of alkyl isocyanides to dialkyl acetylenedicarboxylates was trapped by $\mathrm{N}$-formyl urea to produce highly functionalized dihydropyrimidinones in fairly good yields.
\end{abstract}

KEY WORDS: Alkyl isocyanides, Dialkyl acetylenedicarboxylate, N-Formyl urea, Three-component reaction, Dihydropyrimidinones

\section{INTRODUCTION}

The dihydropyrimidinones (DHPMs) have exhibited interesting and multifaceted pharmacological properties such as antitumor, anti-inflammatory, antiviral, antifungal and antibacterial activity [1-3]. In addition, these compounds have emerged as potential calcium channel blockers, antihypertensive, $\alpha_{1 \mathrm{a}}$-adrenergic antagonists and neuropeptide antagonists [4].

As a consequence, the synthesis of dihydropyrimidinone derivatives has attracted significant attention by Pietro Biginelli [5]. In recent years, new methods for the synthesis of dihydropyrimidinones have been developed by different groups [6-15]. It is still highly valuable to develop newly direct approaches for the efficient synthesis of DHPMs due to the continued importance of these compounds core in organic and medicinal chemistry.

On the other hand, multicomponent reactions (MCRs) have attracted much attention and are particularly useful for the synthesis of chemical and biological important compounds [16-20]. Moreover isocyanide-based multicomponent reactions (IMCRs) by virtue of their synthetic potential, convergent nature and molecular diversity have attracted much attention because of the advantages that they offer in the field of combinatorial chemistry [21-23].

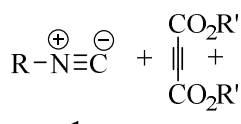

1

\begin{tabular}{l|l}
$\mathbf{1}$ & $\mathrm{R}$ \\
\hline $\mathbf{a}$ & $t-\mathrm{Bu}$
\end{tabular}

b $C y$-Hexyl

c 1,1,3,3-tetra methyl-butyl c $\mathbf{t}$-Bu

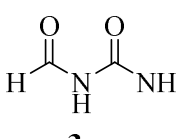

3

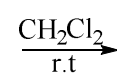

$\mathrm{R}^{\prime}$<smiles></smiles>

\begin{tabular}{l|ll|c}
$\mathbf{4}$ & $\mathrm{R}$ & $\mathrm{R}^{\prime}$ & Yield of $\mathbf{4}(\%)$ \\
\hline $\mathbf{a}$ & $t$-Bu & $\mathrm{Me}$ & 72 \\
$\mathbf{b}$ & $C y$-Hexyl & $\mathrm{Me}$ & 69 \\
$\mathbf{c}$ & $t$-Bu & $\mathrm{Et}$ & 76 \\
$\mathbf{d}$ & $C y$-Hexyl & $\mathrm{Et}$ & 86 \\
$\mathbf{e}$ & $C y$-Hexyl & $t$-Bu & 79 \\
$\mathbf{f}$ & $1,1,3,3$-tetra methyl-butyl & $\mathrm{Me}$ & 75 \\
$\mathbf{g}$ & $1,1,3,3$-tetra methyl-butyl & $\mathrm{Et}$ & 70
\end{tabular}

Scheme 1 . The preparation of substituted dihydropyrimidinones.

*Corresponding author. E-mail: b_mohtat@yahoo.com

This work is licensed under the Creative Commons Attribution 4.0 International License 
Preparation of DHPMs, via multicomponent reactions (MCRs), with high facileness, efficiency and economy in organic chemistry prompted us to synthesize formyl-2-oxo-1,2,3,4tetrahydropyrimidone derivatives. Here in we report an efficient synthetic route to dihydropyrimidinone derivatives (4) using alkyl isocyanides (1) and dialkyl acetylenedicarboxylate (2) in the presence of $\mathrm{N}$-formyl urea (3) (Scheme 1). Compounds 4a-g are all reported for the first time.

\section{EXPERIMENTAL}

General

Melting points were measured on an Electrothermal 9100 apparatus. Elemental analyses for C, $\mathrm{H}$, and $\mathrm{N}$ were performed using a Carlo Erba EA 1108 instrument. EI mass spectra $(70 \mathrm{eV})$ were measured on a Finnigan-MAT- 8430 mass spectrometer. The IR spectra were measured on a Shimadzu IR-460 spectrometer. The ${ }^{1} \mathrm{H}$ and ${ }^{13} \mathrm{C}-\mathrm{NMR}$ spectra were recorded on a Bruker DRX300 Avance instrument with $\mathrm{CDCl}_{3}$ as the solvent at 300 and $75 \mathrm{MHz}$, respectively. Alkyl isocyanides, dialkyl acetylenedicaboxylates and $\mathrm{N}$-formyl urea were obtained from Fluka and were used without further purification.

\section{General procedure for the preparation of pyrimidines 4}

To a stirred solution of $\mathbf{3}(2 \mathrm{mmol})$ and tert-butyl isocyanide $(2 \mathrm{mmol})$ in $10 \mathrm{~mL}$ of $\mathrm{CH}_{2} \mathrm{Cl}_{2}$, dimethyl acetylenedicarboxylate (DMAD) $(2 \mathrm{mmol})$ was added dropwise and the reaction mixture was then allowed to warm to room temperature and stand for $24 \mathrm{~h}$. The solvent was removed under partial vacuum reduced pressure and oily residue was purified by preparative TLC on silica gel (Merck silica gel DCFertigplatten 60/Kieselgur $\mathrm{F}_{254}$ ) $20 \times 20 \mathrm{~cm}$ glass plates using n-hexane-EtOAc (1:1) as eluent.

Dimethyl 6-(tert-butylamino)-3-formyl-2-oxo-1,2,3,4-tetrahydropyrimidine-4,5-dicarboxylate (4a). Yellow powder, $\left(0.45 \mathrm{~g}, 72 \%\right.$ yield) $\mathrm{mp} 157-159{ }^{\circ} \mathrm{C}$; IR $(\mathrm{KBr}) v_{\max } 3423,2956,2854$, $1735,1699,1461,1262,1188,1025,965,803 \mathrm{~cm}^{-1} ;{ }^{1} \mathrm{H} \mathrm{NMR}\left(\mathrm{CDCl}_{3}, 300 \mathrm{MHz}\right) \delta 9.34(1 \mathrm{H}, \mathrm{s}$, $\mathrm{CHO}), 8.77(1 \mathrm{H}$, brs, $\mathrm{NH}), 6.88(1 \mathrm{H}$, brs, $\mathrm{NH}), 5.91(1 \mathrm{H}, \mathrm{s}, \mathrm{CH}), 3.77\left(3 \mathrm{H}, \mathrm{s}, \mathrm{OCH}_{3}\right), 3.68(3 \mathrm{H}$, $\left.\mathrm{s}, \mathrm{OCH}_{3}\right), 1.30\left(9 \mathrm{H}, \mathrm{s}, 3 \mathrm{CH}_{3}\right) ;{ }^{13} \mathrm{C} \mathrm{NMR}\left(\mathrm{CDCl}_{3}, 75 \mathrm{MHz}\right) \delta 170.0(\mathrm{CHO}), 167.7(\mathrm{C}=\mathrm{O}$ esrer $)$, $160.6\left(C=\mathrm{O}\right.$ ester), $151.0\left(\mathrm{~N}_{2} C=\mathrm{O}\right), 147.0\left(\mathrm{CN}_{2}\right), 71.4(\mathrm{C}), 68.1(C-\mathrm{N}), 53.7\left(\mathrm{OCH}_{3}\right), 52.0$ $\left(\mathrm{OCH}_{3}\right), 49.5(\mathrm{CH}), 29.6\left(3 \mathrm{CH}_{3}\right)$; EI-MS m/z (rel. int.): $313\left[\mathrm{M}^{+}\right](13), 212(54), 195(72), 83$ (78), 57 (100). Anal. C 49.87\%, H 6.10\%, N 13.40\%, calcd. for $\mathrm{C}_{13} \mathrm{H}_{19} \mathrm{~N}_{3} \mathrm{O}_{6}, \mathrm{C} 49.84 \%, \mathrm{H}$ $6.11 \%, \mathrm{~N} 13.41 \%$.

Dimethyl 6-(cyclohexylamino)-3-formyl-2-oxo-1,2,3,4-tetrahydropyrimidine-4,5-dicarboxylate (4b). Yellow powder, (0.46 g, $69 \%$ yield), mp 165-167 ${ }^{\circ} \mathrm{C}$; IR (KBr) $v_{\max } 3421,2932,2855$, $1739,1693,1436,1262,1165,1026,953,755 \mathrm{~cm}^{-1} ;{ }^{1} \mathrm{H} \mathrm{NMR}\left(\mathrm{CDCl}_{3}, 300 \mathrm{MHz}\right) \delta 9.44(\mathrm{~s}, 1 \mathrm{H}$, $\mathrm{CHO}), 8.81(1 \mathrm{H}$, brs, $\mathrm{NH}), 8.10(1 \mathrm{H}$, brs, $\mathrm{NH}), 5.84(1 \mathrm{H}, \mathrm{s}, \mathrm{CH}), 3.78\left(3 \mathrm{H}, \mathrm{s}, \mathrm{OCH}_{3}\right), 3.78(1 \mathrm{H}$, $\mathrm{s}, \mathrm{CH}), 3.74\left(3 \mathrm{H}, \mathrm{s}, \mathrm{OCH}_{3}\right), 1.21-1.62\left(10 \mathrm{H}, \mathrm{m}, 5 \mathrm{CH}_{2}\right) ;{ }^{13} \mathrm{C} \mathrm{NMR}\left(\mathrm{CDCl}_{3}, 75 \mathrm{MHz}\right) \delta 169.7$ $(C \mathrm{HO}), 167.1(C=\mathrm{O}$ ester $), 160.5(C=\mathrm{O}$ ester $), 151.8\left(\mathrm{~N}_{2} C=\mathrm{O}\right), 149.4\left(C \mathrm{~N}_{2}\right), 70.6(\mathrm{C}), 53.7$ $\left(\mathrm{OCH}_{3}\right), 52.3\left(\mathrm{OCH}_{3}\right), 50.0(\mathrm{CHN}), 49.8(\mathrm{CH}), 33.6\left(\mathrm{CH}_{2}\right), 25.0\left(\mathrm{CH}_{2}\right), 24.3\left(\mathrm{CH}_{2}\right), 24.2\left(\mathrm{CH}_{2}\right)$; EI-MS m/z (rel. int.): 339 [M ] (21), 221 (100), 212 (84), 57 (74), 41 (27). Anal. C, 52.89\%, H $6.18 \%, \mathrm{~N} 12.47 \%$, calcd. for $\mathrm{C}_{15} \mathrm{H}_{21} \mathrm{~N}_{3} \mathrm{O}_{6}, \mathrm{C} 53.09 \%$, H $6.24 \%$, N $12.38 \%$.

Diethyl 6-(tert-butylamino)-3-formyl-2-oxo-1,2,3,4-tetrahydropyrimidine-4,5-dicarboxylate (4c). Yellow powder, (052 g, 76\% yield), mp 153-155 ${ }^{\circ} \mathrm{C}$; IR (KBr) $v_{\max } 3411,2938,2855$, 1737, 1686, 1440, 1199, 1141, 1023, 953, $708 \mathrm{~cm}^{-1} ;{ }^{1} \mathrm{H} \mathrm{NMR}\left(\mathrm{CDCl}_{3}, 300 \mathrm{MHz}\right) \delta 9.33(1 \mathrm{H}, \mathrm{s}$, $\mathrm{CHO}), 8.78(1 \mathrm{H}$, brs, $\mathrm{N} H), 6.95(1 \mathrm{H}$, brs, $\mathrm{N} H), 5.86(1 \mathrm{H}, \mathrm{s}, \mathrm{CH}), 4.08-4.38\left(4 \mathrm{H}, \mathrm{m}, 2 \mathrm{OCH}_{2}\right)$, 
$1.45\left(9 \mathrm{H}, \mathrm{s} .3 \mathrm{CH}_{3}\right), 1.30\left(3 \mathrm{H}, \mathrm{t}, J=7.1 \mathrm{~Hz}, \mathrm{CH}_{3}\right), 1.23\left(3 \mathrm{H}, \mathrm{t}, J=7.1 \mathrm{~Hz}, \mathrm{CH}_{3}\right) ;{ }^{13} \mathrm{C} \mathrm{NMR}$ $\left(\mathrm{CDCl}_{3}, 75 \mathrm{MHz}\right) \delta 169.6(\mathrm{CHO}), 167.5(C=\mathrm{O}$ ester $), 160.7(C=\mathrm{O}$ ester $), 150.9\left(\mathrm{~N}_{2} C=\mathrm{O}\right), 150.4$ $\left(\mathrm{CN}_{2}\right), 72.6(\mathrm{C}), 61.8\left(\mathrm{OCH}_{2}\right), 59.6\left(\mathrm{OCH}_{2}\right), 52.2(\mathrm{C}-\mathrm{N}), 49.7(\mathrm{CH}), 30.9\left(3 \mathrm{CH}_{3}\right) ; 14.55\left(\mathrm{CH}_{3}\right)$, $13.95\left(\mathrm{CH}_{3}\right)$; EI-MS $m / z$ (rel. int.): $341\left[\mathrm{M}^{+}\right](10), 241$ (70), 223 (82), 83 (68), 57 (100). Anal. C, $52.82 \%$, H 6.70\%, N $12.26 \%$, calcd. for $\mathrm{C}_{15} \mathrm{H}_{23} \mathrm{~N}_{3} \mathrm{O}_{6}$. C $52.78 \%$, H 6.79\%, N $12.31 \%$.

Diethyl 6-(cyclohexylamino)-3-formyl-2-oxo-1,2,3,4-tetrahydropyrimidine-4,5-dicarboxylate (4d). Yellow powder $\left(0.63 \mathrm{~g}, 86 \%\right.$ yield), $\mathrm{mp} 150-152{ }^{\circ} \mathrm{C}$; IR $(\mathrm{KBr}) v_{\max } 33430,2944,2856$, $1738,1698,1449,1370,1199,1025,954,750 \mathrm{~cm}^{-1} ;{ }^{1} \mathrm{H} \mathrm{NMR}\left(\mathrm{CDCl}_{3}, 300 \mathrm{MHz}\right) \delta 9.35(1 \mathrm{H}, \mathrm{s}$, $\mathrm{CHO}), 8.75(1 \mathrm{H}$, brs, $\mathrm{N} H), 8.00(1 \mathrm{H}$, brs, $\mathrm{N} H), 5.87(1 \mathrm{H}, \mathrm{s}, \mathrm{CH}), 4.03-4.32\left(4 \mathrm{H}, \mathrm{m}, 2 \mathrm{OCH}_{2}\right)$, 3.42-3.49 (1H, m, CH), $1.32\left(3 \mathrm{H}, \mathrm{t}, J=7.1 \mathrm{~Hz}, \mathrm{CH}_{3}\right), 1.25\left(3 \mathrm{H}, \mathrm{t}, J=7.1 \mathrm{~Hz}, \mathrm{CH}_{3}\right), 1.15-1.46$, $\left(10 \mathrm{H}, \mathrm{m}, \mathrm{CH}_{2}\right) ;{ }^{13} \mathrm{C} \mathrm{NMR}\left(\mathrm{CDCl}_{3}, 75 \mathrm{MHz}\right) \delta 169.9$ (CHO), $167.5(\mathrm{C}=\mathrm{O}$ ester $), 160.7(\mathrm{C}=\mathrm{O}$ ester), $151.8\left(\mathrm{~N}_{2} C=\mathrm{O}\right), 149.8\left(\mathrm{CN}_{2}\right), 70.7(\mathrm{C}), 61.8\left(\mathrm{OCH}_{2}\right), 59.4\left(\mathrm{OCH}_{2}\right), 50.2(C \mathrm{HN}), 49.8$ $(\mathrm{CH}), 33.6\left(\mathrm{CH}_{2}\right), 29.7\left(\mathrm{CH}_{2}\right), 25.0\left(\mathrm{CH}_{2}\right), 24.3\left(\mathrm{CH}_{2}\right), 24.2\left(\mathrm{CH}_{2}\right), 14.5\left(\mathrm{CH}_{3}\right), 13.9\left(\mathrm{CH}_{3}\right)$; EIMS $m / z$ (rel. int.): $367\left[\mathrm{M}^{+}\right](5), 241$ (67), $223(82), 57(100), 41(68)$. Anal. C 55.67\%, H $6.79 \%$, N $11.40 \%$, calcd. for $\mathrm{C}_{17} \mathrm{H}_{25} \mathrm{~N}_{3} \mathrm{O}_{6}, \mathrm{C} 55.58 \%$, H 6.86\%, N $11.44 \%$.

Di-tert-butyl

6-(cyclohexylamino)-3-formyl-2-oxo-1,2,3,4-tetrahydropyrimidine-4,5dicarboxylate (4e). Yellow powder $\left(0.67 \mathrm{~g}, 79 \%\right.$ yield), mp $167-169{ }^{\circ} \mathrm{C}$; IR (KBr) $v_{\max } 3419$, 2934, 2856, 1733, 1698, 1437, 1255, 1150, 1022, 953, $707 \mathrm{~cm}^{-1} ;{ }^{1} \mathrm{H} \mathrm{NMR}\left(\mathrm{CDCl}_{3}, 300 \mathrm{MHz}\right) \delta$ $9.32(\mathrm{~s}, 1 \mathrm{H}, \mathrm{CHO}), 8.64(1 \mathrm{H}$, brs, $\mathrm{NH}), 7.64(1 \mathrm{H}$, brs, $\mathrm{N} H), 5.65(1 \mathrm{H}, \mathrm{s}, \mathrm{CH}), 3.30-3.36(1 \mathrm{H}, \mathrm{m}$, $\mathrm{CH}), 1.51\left(9 \mathrm{H}, \mathrm{s}, 3 \mathrm{CH}_{3}\right), 1.43\left(9 \mathrm{H}, \mathrm{s}, 3 \mathrm{CH}_{3}\right), 1.23-1.32\left(10 \mathrm{H}, \mathrm{m}, \mathrm{CH}_{2}\right) ;{ }^{13} \mathrm{C} \mathrm{NMR}\left(\mathrm{CDCl}_{3}, 75\right.$ MHz) $\delta 169.2(C H O), 167.3\left(C=\mathrm{O}\right.$ ester), $160.8(C=\mathrm{O}$ ester $), 151.9\left(\mathrm{~N}_{2} C=\mathrm{O}\right), 149.4\left(C_{2}\right), 82.3$ $(C-\mathrm{O}), 79.8(\mathrm{C}-\mathrm{O}), 72.4(\mathrm{C}), 50.8(\mathrm{CHN}), 50.4(\mathrm{CH}), 27.9\left(3 \mathrm{CH}_{3}\right), 27.7\left(3 \mathrm{CH}_{3}\right)$; EI-MS $\mathrm{m} / z$ (rel. int.): $423\left[\mathrm{M}^{+}\right]$(9), 325 (67) 296 (76), 57 (88), 41 (45). Anal. C 59.46\%, H 7.78\%, N 9.83\%, $\mathrm{C}_{21} \mathrm{H}_{33} \mathrm{~N}_{3} \mathrm{O}_{6}$, calcd. for C $59.56 \%$, H 7.85\%, N 9.92\%.

Dimethyl 6-(2,4,4-trimethylpentan-2-ylamino)-3-formyl-2-oxo-1,2,3,4-tetrahydropyrimidine4,5-dicarboxylate (4f). Yellow powder, $\left(0.55 \mathrm{~g}, 75 \%\right.$ yield), $\mathrm{mp} 166-168{ }^{\circ} \mathrm{C}$; IR (KBr) $v_{\max }$ $3423,2954,2854,1742,1703,1436,1217,1149,1023,953,708 \mathrm{~cm}^{-1} ;{ }^{1} \mathrm{H}$ NMR $\left(\mathrm{CDCl}_{3}, 300\right.$ MHz) $\delta 9.32(1 \mathrm{H}, \mathrm{s}, \mathrm{CHO}), 8.84(1 \mathrm{H}$, brs, $\mathrm{NH}), 8.70(1 \mathrm{H}$, brs, $\mathrm{NH}), 5.74(1 \mathrm{H}, \mathrm{s}, \mathrm{CH}), 3.74(3 \mathrm{H}$, $\left.\mathrm{s}, \mathrm{OCH}_{3}\right), 3.69\left(3 \mathrm{H}, \mathrm{s}, \mathrm{OCH}_{3}\right), 1.75-1.60\left(2 \mathrm{H}, \mathrm{m}, \mathrm{CH}_{2}\right), 1.52\left(3 \mathrm{H}, \mathrm{s}, \mathrm{CH}_{3}\right), 1.50\left(3 \mathrm{H}, \mathrm{s}, \mathrm{CH}_{3}\right)$, $1.00\left(9 \mathrm{H}, \mathrm{s}, 3 \mathrm{CH}_{3}\right) ;{ }^{13} \mathrm{C} \mathrm{NMR}\left(\mathrm{CDCl}_{3}, 75 \mathrm{MHz}\right) \delta 170.0(\mathrm{CHO}), 167.9(\mathrm{C}=\mathrm{O}$ ester $), 160.6(\mathrm{C}=\mathrm{O}$ ester), $151.1\left(\mathrm{~N}_{2} C=\mathrm{O}\right), 150.3\left(\mathrm{CN}_{2}\right), 71.8(\mathrm{C}), 55.9(\mathrm{CN}), 53.4\left(\mathrm{OCH}_{3}\right), 53.2\left(\mathrm{OCH}_{3}\right), 51.0$ $\left(\mathrm{CH}_{2}\right), 49.4(\mathrm{CH}), 34.6\left(\mathrm{CH}_{3}\right), 31.8(\mathrm{C}), 31.4\left(3 \mathrm{CH}_{3}\right), 31.3\left(\mathrm{CH}_{3}\right)$; EI-MS $m / z$ (rel. int.): 369 $\left[\mathrm{M}^{+}\right]$(17), 251 (83), 211 (43), 128 (69), 57 (96). Anal. C 55.20\%, H 7.40\%, N 11.29\%, calcd. for $\mathrm{C}_{17} \mathrm{H}_{27} \mathrm{~N}_{3} \mathrm{O}_{6}$, C 55.27\%, H 7.37\%, N $11.37 \%$.

Diethyl 6-(2,4,4-trimethylpentan-2-ylamino)-3-formyl-2-oxo-1,2,3,4-tetrahydropyrimidine-4,5dicarboxylate $(\mathbf{4 g})$. Yellow powder, $\left(0.55 \mathrm{~g}, 70 \%\right.$ yield), mp $172-174{ }^{\circ} \mathrm{C}$; IR $(\mathrm{KBr}) v_{\max } 3421$, 2957, 2857, 1746, 1698, 1473, 1254, 1162, 1026, 690, $780 \mathrm{~cm}^{-1} ;{ }^{1} \mathrm{H} \mathrm{NMR}\left(\mathrm{CDCl}_{3}, 300 \mathrm{MHz}\right) \delta$ $9.53(1 \mathrm{H}, \mathrm{s}, \mathrm{CHO}), 9.34(1 \mathrm{H}$, brs, $\mathrm{N} H), 9.31(1 \mathrm{H}$, brs, $\mathrm{N} H), 5.70(1 \mathrm{H}, \mathrm{s}, \mathrm{CH}), 4.14-4.38(4 \mathrm{H}, \mathrm{m}$, $\left.2 \mathrm{OCH}_{2}\right), 1.78-1.72\left(2 \mathrm{H}, \mathrm{m}, \mathrm{CH}_{2}\right), 1.54\left(3 \mathrm{H}, \mathrm{s}, \mathrm{CH}_{3}\right), 1.51\left(3 \mathrm{H}, \mathrm{s}, \mathrm{CH}_{3}\right), 1.01\left(9 \mathrm{H}, \mathrm{s}, 3 \mathrm{CH}_{3}\right), 0.91$ $\left(3 \mathrm{H}, \mathrm{t}, J=7.1 \mathrm{~Hz}, \mathrm{CH}_{3}\right), 0.86\left(3 \mathrm{H}, \mathrm{t}, J=7.1 \mathrm{~Hz}, \mathrm{CH}_{3}\right) ;{ }^{13} \mathrm{C} \mathrm{NMR}\left(\mathrm{CDCl}_{3}, 75 \mathrm{MHz}\right) \delta 170.2$ (CHO), $167.6(C=\mathrm{O}$ ester $), 160.5(C=\mathrm{O}$ ester $), 150.8\left(\mathrm{~N}_{2} C=\mathrm{O}\right), 150.3\left(C \mathrm{~N}_{2}\right), 71.7(C \mathrm{H}), 61.2$ $\left(\mathrm{OCH}_{2}\right), 56.2(\mathrm{CN}), 59.5\left(\mathrm{OCH}_{2}\right), 53.3\left(\mathrm{CH}_{2}\right), 49.9(\mathrm{CH}), 31.7\left(\mathrm{CH}_{3}\right), 31.6(\mathrm{C}), 31.3\left(3 \mathrm{CH}_{3}\right)$, $31.0\left(\mathrm{CH}_{3}\right), 14.7\left(\mathrm{CH}_{3}\right), 14.4\left(\mathrm{CH}_{3}\right)$; EI-MS m/z (rel. int.): $397\left[\mathrm{M}^{+}\right]$(7), 269 (39), 251 (52), 83 (96), 57 (94). Anal. C 57.33\%, H 7.78\%, N 10.64\%, calcd. for $\mathrm{C}_{19} \mathrm{H}_{31} \mathrm{~N}_{3} \mathrm{O}_{6}, \mathrm{C} 57.41 \%$, $\mathrm{H} 7.86 \%$, N $10.57 \%$. 


\section{RESULTS AND DISCUSSION}

Herein, a number of new 3-formyl-2-oxo-1,2,3,4-tetrahydropyrimidine derivatives 4 were prepared at room temperature reaching completion in 24 hours. The structural assignments of the desired dialkyl 6-(alkylamino)-3-formyl-2-oxo-1,2,3,4-tetrahydropyrimidine-4,5-dicarboxylate derivatives 4a-g were made on the basis of their ${ }^{1} \mathrm{H}$ and ${ }^{13} \mathrm{C}$ NMR spectra which are supported by their IR and mass spectrometry as well as elemental analysis.

The ${ }^{1} \mathrm{H}$ NMR spectrum of $\mathbf{4 a}$ exhibited five sharp singlets for tert-butyl $(\delta=1.30 \mathrm{ppm})$, methoxy $(\delta=3.68$ and $3.77 \mathrm{ppm})$, methine $(\delta=5.91 \mathrm{ppm})$ and aldehyde proton $(9.34 \mathrm{ppm})$ respectively. The NHs proton resonance at $(\delta=6.88,8.77 \mathrm{ppm})$ disappeared after addition of $\mathrm{D}_{2} \mathrm{O}$ to the $\mathrm{CDCl}_{3}$ solution of $4 \mathbf{a}$. The ${ }^{1} \mathrm{H}$ decoupled ${ }^{13} \mathrm{C}$ NMR spectrum of $4 \mathbf{a}$ showed eleven distinct resonances in agreement with the proposed structure. The presence of amido and amino groups at one end of the double bond leads to polarization of the olefinic system. The $\alpha$-carbon atom of this polarized system appears at $\delta=147.0$, while and the $\beta$-carbon at $\delta=71.4 \mathrm{ppm}$. Partial assignments of these resonances are given in the experimental section. The ${ }^{1} \mathrm{H}$ NMR spectra of compounds $\mathbf{4 b} \mathbf{- 4 g}$ are similar to that of compound $\mathbf{4 a}$, except for the signals of the alkyl and ester moiety. TLC results of reaction mixture and also ${ }^{1} \mathrm{H}$ and ${ }^{13} \mathrm{C}$ NMR spectra of compound 4a-g showed that only one steroisomer is produced.

A possible explanation is proposed in Scheme 2. On the basis of the well-established chemistry of isocyanides [24, 25], nucleophilic addition of alkyl isocyanide $\mathbf{1}$ to dialkyl acetylenedicarboxylate $\mathbf{2}$ leads to zwitterion $\mathbf{5}$ which is protonated by $\mathrm{NH}$ acid to give $\mathbf{6}$. This intermediate is attacked by anion 7 to produce ketenimine 8. 1, 3-Proton transfer and subsequent cyclization forms products 4 (Scheme 2). The reaction of alkyl isocyanides (1) and $\mathrm{N}$-formyl urea (3) did not lead to a remarkable product via TLC monitoring.

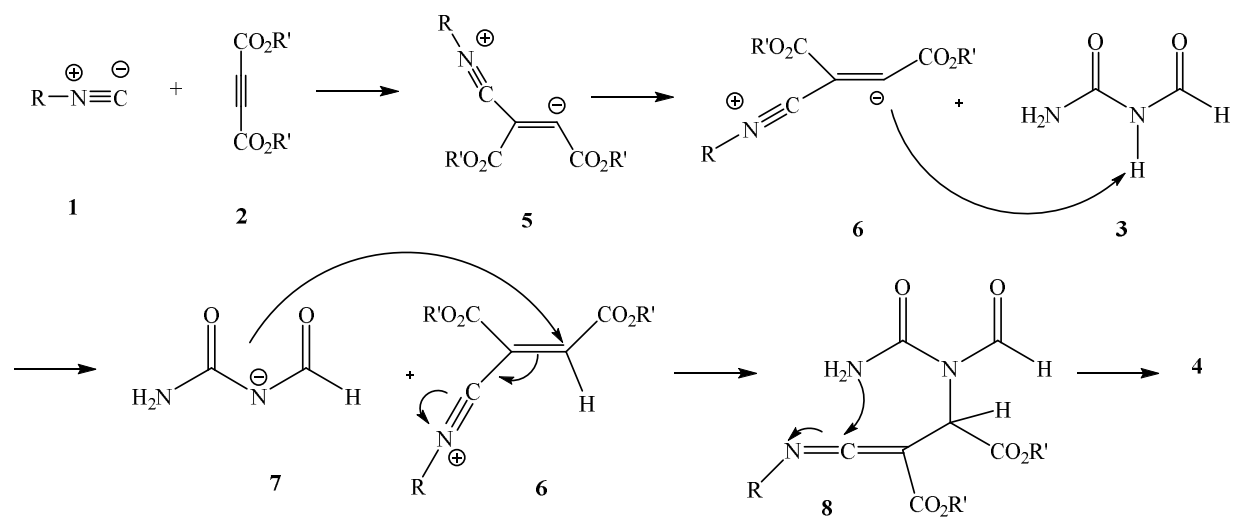

Scheme 2. Proposed mechanism.

\section{CONCLUSION}

In conclusion, a new method for the preparation of highly functionalized dihydropyrimidinone has been demonstrated. The present method carries the advantage of performing under neutral conditions and also the starting materials can be mixed without any activation or modification. 


\section{REFERENCES}

1. Kappe, C.O. Biologically active dihydropyrimidones of the Biginelli-type a literature survey. Eur. J. Med. Chem. 2000, 35, 1043-1052.

2. Chitra, S.; Devanathan, D.; Pandiarajan, K. Synthesis and in vitro microbiological evaluation of novel 4-aryl-5-isopropoxycarbonyl-6-methyl-3,4-dihydropyrimidinones. Eur. J. Med. Chem. 2010, 45, 367-371.

3. Chhillar, A.K.; Arya, P.; Mukherjee, C. Microwave-assisted synthesis of antimicrobial dihydropyridines and tetrahydropyrimidin-2-ones: Novel compounds against aspergillosis. Bioorg. Med. Chem. 2006, 14, 973-981.

4. (a) Atwal, K.S.; Swanson, B.N.; Unger, S.E.; Floyed D.M.; Moreland, S.; Hedberg, A.; O'Reilly, B.C. Dihydropyrimidine calcium channel blockers. 3. 3-Carbamoyl-4-aryl-1,2,3,4tetrahydro-6-methyl-5-pyrimidinecarboxylic acid esters as orally effective antihypertensive agents. J. Med. Chem. 1991, 34, 806-811. (b) Atwal, K.S.; Roonyak, G.C.; O'Reilly, B.C.; Schwartz, A. Substituted 1,4-dihydropyrimidines. 3. Synthesis of selectively functionalized 2-hetero-1,4-dihydropyrimidines. J. Org. Chem. 1989, 54, 5898-5907.

5. Biginelli, P. Synthesis of 3,4-dihydropyrimidin-2(1H)-ones. Gazz. Chim. Ital. 1893, 23, 360416.

6. Qu, H.; Li, X.; Mo, F.; Lin, X. Efficient synthesis of dihydropyrimidinones via a threecomponent Biginelli-type reaction of urea, alkylaldehyde and arylaldehyde. Beilstein J. Org. Chem. 2013, 9, 2846-2851.

7. Mohamed, K.S.; Soliman, M.A.; El-Remaily, M.A.A.; Abdel-Ghany, H. Eco-friendly synthesis of pyrimidine and dihydropyrimidinone derivatives under solvent free condition and their anti-microbial activity. Chem. Sci. J. 2013, 7, 1-11.

8. Karade N.H.; Sathe, M.; Kaushik, P.M. Synthesis of 4-aryl substituted 3,4dihydropyrimidinones using silica-chloride under solvent free conditions. Molecules 2007, $12,1341-1351$.

9. Han, X.; Xu, F.; Luo, Y.; Shen, Q. An efficient one-pot synthesis of dihydropyrimidinones by a samarium diiodide catalyzed Biginelli reaction under solvent-free conditions. Eur. J. Org. Chem. 2005, 2005, 1500-1503.

10. Lin, H. X.; Zhao, Q. J.; Xu, B.; Wang, X. H. A green synthesis of dihydropyrimidinones by Biginelli reaction over Nafion-H catalyst. Chin. Chem. Lett. 2007, 18, 502-504.

11. Alikarami, M.; Moradi, R. Benzyltriphenylphosphonium chloride: A efficient catalyst for one-pot synthesis of dihydropyrimidinones/thiones under solvent-free conditions. Lett. Org. Chem. 2015, 12, 560-512.

12. Roy, S.R.; Jadhavar, P.S.; Seth, K.; Sharma, K.K. Chakraborti, A.K. Organocatalytic application of ionic liquids: [bmim][MeSO4] as a recyclable organocatalyst in the multicomponent reaction for the preparation of dihydropyrimidinones and thiones. Synthesis 2011, 14, 2261-2267.

13. Chechina, N.V.; Zubar, V.V.; Omelchenko, I.V.; Kolos, N.N. One-pot synthesis of new derivatives of 3,4-dihydropyrimidinone, and substituted imidazolin-2-ones. Arkivoc, 2015, 2015, 293-304.

14. Hekmatshoar, R.; Heidari, M.; Heravi, M.M.; Baghernejad B. Efficient sodiumselenatecatalyzed synthesis of 3,4-dihydro-2(1H)-pyrimidinones and -thiones under solvent-free conditions. Bull. Chem. Soc. Ethiop. 2009, 23, 141-144.

15. Kathing, C.; Rani, J.W.S.; Singh, N.G.; Tumtin, S.; Nongrum, R.; Nongkhlaw, R. One-pot synthesis of 3,4-dihydropyrimidin-2(1H)-ones catalysed by cupric acetate under solvent-free conditions. J. Chin. Chem. Soc. 2014, 61, 1254-1258.

16. Trost, B.M. On inventing reactions for atom economy. Acc. Chem. Res. 2002, 35, 695-705.

17. Zhu, J.; Bienayme, H. Multicomponent Reactions, Wiley-VCH: Weinheim, Germany; 2005; p 76. 
18. Dömling, A. Recent developments in isocyanide based multicomponent reactions in applied chemistry. Chem. Rev. 2006, 106, 17-89.

19. Tejedor, D.; Garcia-Tellado, F. Chemo-differentiating $\mathrm{ABB}^{\prime}$ multicomponent reactions: Privileged building blocks. Chem. Soc. Rev. 2007, 36, 484-491.

20. Müller, T.J.J. Multicomponent reactions. Beilstein J. Org. Chem. 2011, 7, 960-961.

21. Ugi, I. Werner, B. Dömling, A. The chemistry of isocyanides, their multicomponent reactions and their libraries. Molecules 2003, 8, 53-66.

22. Ugi, I. From isocyanides via four-component condensations to antibiotic synthesis. Angew. Chem. Int. Engl. Ed. 1982, 21, 810-819.

23. Ramazani, A.; Shaghaghi,Z.; Aghahosseini, H.; Asiabi, P.A.; Joo, S.W. Silica nanoparticles as a highly efficient catalyst for the one-pot synthesis of sterically congested 2(dibenzylamino)-2-aryl acetamide derivatives from by phthaldehyde isomers, isocyanides and dibenzylamine. Bull. Chem. Soc. Ethiop. 2016, 30, 413-420

24. Walborsky, H.M.; Periasamy, M.P. in The Chemistry of Functional Groups, Chapter 20, Patai, S.; Rappoport, Z. (Eds.), Wiley: New York; 1983; p 835.

25. Marcaccini, S.; Torroba, T. The use of isocyanides in heterocylic synthesis. Org. Prep. Proced. Int. 1993, 25, 141-208. 